\title{
A cohort analysis of breast cancer, uterine corpus cancer, and childbearing pattern in Norwegian women
}

\author{
Steinar Tretli, Tor Haldorsen
}

\begin{abstract}
Study objective-The aim was to study the influence of childbearing pattern on the incidence of breast cancer and uterine corpus cancer.
\end{abstract}

Design-This was an ecological study of birth cohorts of women.

Setting-The study was population based, involving the whole of Norway.

Participants-The participants were Norwegian women born between 1890 and 1944.

Measurements and main results-Age specific fertility rates and age specific incidence rates for different birth cohorts were analysed by an age-cohort-period model where quantitative indices of the childbearing pattern substituted the cohort component. The 1890-94 birth cohort had the most favourable childbearing pattern with regard to the risk of breast cancer as well as uterine corpus cancer. The least favourable pattern was in the 1910-14 cohort for breast cancer and the $1940-44$ cohort for uterine corpus cancer. In the analysis it is estimated that about $15 \%$ of the increase in incidence of breast cancer from 1955 to 1984 may be attributed to changes in the childbearing pattern of the cohorts under study. For cancer of the uterine corpus the corresponding fraction is about $27 \%$.

Conclusions-The study reveals that changes in childbearing pattern may explain a certain fraction of the observed increase in breast and uterine corpus cancer in Norway in the last 30 years, but the largest fraction must be accounted for by other factors.

Most studies of the effect of pregnancy on the risk of breast cancer indicate a protective effect of high parity. A positive association between the age at first pregnancy and risk of breast cancer has been found, but when taking parity into account the strength of this association has not been clarified. ${ }^{1}$ In a recent paper by Kvalle and $\mathrm{Heuch}^{2}$ it is suggested that the relation between the age at pregnancy and breast cancer risk may be more complex than previously assumed. They found that the age at last birth was positively associated with breast cancer incidence.

The childbearing pattern also seems to have an influence on the incidence of uterine cancer. Studies have shown a higher risk of uterine corpus cancer among nulliparous women ${ }^{3}$ and an inverse relation with parity. 4

The age adjusted incidence of breast cancer in
Norway increased by 50\% from 1955 to 1984 and of uterine corpus cancer by $75 \%$. A pertinent question is whether a change in childbearing pattern could "account for" this increase in incidence. In the present study we shall try to elucidate this problem by applying quantitative indices of the childbearing pattern for each birth cohort in an age-cohort-period model for incidence of these two types of cancer. The plan for this study originally also included ovarian cancer. Unfortunately, a shift in the diagnosis criteria for ovarian cancer in our registry in the late 60 s made this type of analysis unsuitable.

\section{Methods}

MATERIAL

All births and age of the mothers at childbearing have been registered for all female residents of Norway since 1845 by the Central Bureau of Statistics. ${ }^{5}$ This gave us the opportunity to define four variables describing the indices of childbearing pattern for each of the five year birth cohorts from 1890-94 to $1940-44$ :

NC19: Number of children born per woman aged 15-19 years

NC29: Number of children born per woman aged 20-29 years

NC39: Number of children born per woman aged 30-39 years

NC49: Number of children born per woman aged 40-49 years

Children born to women older than the age at cancer we are studying minus 5 years are not included in NC39 and NC49.

NC19 will be a variable indicating the cohort's tendency to have the first child in early life, while NC49 indicates the tendency to have children late in the fertile period of life. Variations in NC29 and NC39 between different cohorts will mainly indicate differences in parity.

All new cases of cancer have been registered in Norway since 1953 . Incidence data for the period 1955-84 will be applied in this study. Ninety seven per cent of the breast cancer cases and $99^{\circ}$ o of the uterine corpus cancer cases were histologically confirmed.

Only the age group 30-69 years was considered in this study. This is because we were not sure of the appropriateness of including older age groups in such a computation.

ANALYSES

Let $D_{i j k} ; i=1,2, \ldots, 7 ; k=1,2 \ldots, 5 ; j=7-i+k$; be number of observed cases in age group $i$ ( 5 year 
age groups 35-69 years), birth cohort $j$ (5 year cohort groups 1890-1944) and period of diagnosis $k$ (five periods 1955-84). At first we shall assume that $D_{i j k}$ is Poisson distributed with mean $\exp \left(\xi+\alpha_{j}+\pi_{k}+\beta_{j}+\log \left[N_{i j}\right]\right)$ where $\xi$ is the constant term, $\alpha_{j}$ is the age effect, $\pi_{k}$ is the period effect and $\beta_{j}$ the cohort effect and childbearing pattern and $\mathrm{N}_{\mathrm{ij}}$ is the number at risk in age group $i$ and cohort j. ${ }^{6}$ Estimation and testing were done by using GLIM 3.77. This model, named "model 1", is thought of as a reference model. An acceptable goodness of fit appraised by the deviance of this model has been the premise for adapting the data to the model where the cohort effect $\beta_{j}$ is substituted by $\gamma_{1} \star \mathrm{NC1} 9_{j}+\gamma_{2} \star \mathrm{NC} 29_{j}+\gamma_{3} \mathrm{NC} 39_{j}+\gamma_{4} \star \mathrm{NC} 49_{j}$. $\gamma_{1}, \gamma_{2}, \gamma_{3}, \gamma_{4}$ are the coefficients of the variables describing childbearing pattern. We name this last model "model 2 ".

\section{Results}

Figure 1 shows the changes in the four variables we have used to describe the childbearing patterns by birth cohort from 1890-94 to $1940-44$. The number of children born per year by women 15-19 years of age was almost constant for the cohorts before 1920-24. Later cohorts show an increase up to a level in the cohort 1940-44 that is almost three times higher than in the cohort 1920-24. The number of children born per year by women over 40 years of age decreased steadily by cohorts later than 1905-09 and today only a few women have pregnancies over 40 years of age. Most of the children are born to women between 20 and 29 years of age, with the smallest number in the 1905-14 cohorts. The number of children born per year by women 30-39 years of age has been decreasing from the 1890-94 cohort to the 1900-04 cohort and from the 1910-14 cohort to the 1940-44 cohort.

Figure 2 shows the observed incidence of breast cancer by age and birth cohort. The figure shows the well known hook first described by Clemmesen $^{7}$ at age $50-54$. However, there is a

Figure 1 Age specific birth rate by women's birth cohort.

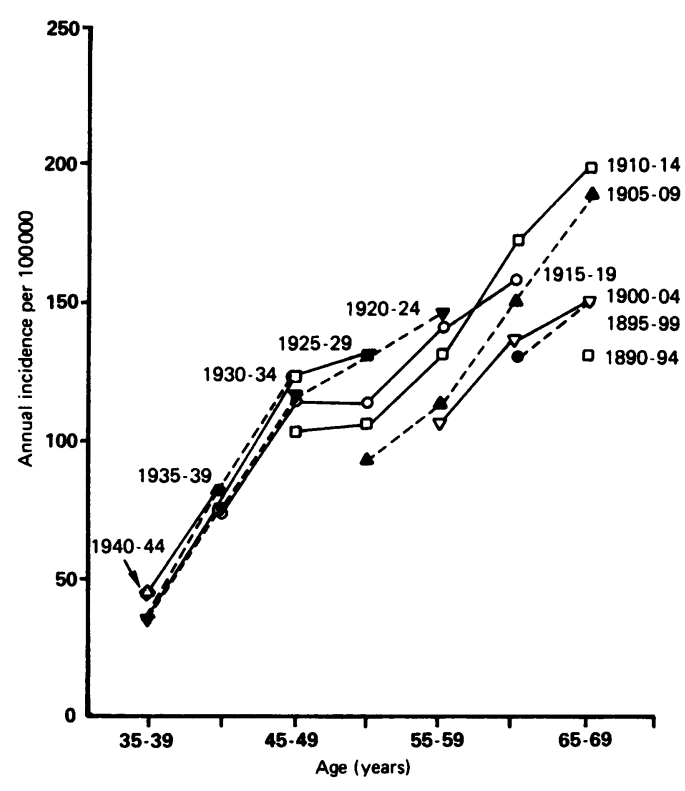

Figure 2 Incidence of breast cancer by age and birth cohort.

tendency for the hook to be less pronounced in the younger cohorts. The increase in breast cancer incidence by age is somewhat greater in the age-groups below the mean menopause age than in the age groups above.

Fitting model 1 to the data, we have an acceptable fit with a deviance of 17.8 with 15 degrees of freedom ( 15 is the expected deviance). By substituting the general cohort parameters by the four quantitative variables describing chidbearing pattern, we obtained for this model (model 2) a deviance of 20.4 with 20 degrees of freedom. The difference in deviance between these two models ( 2.6 with 5 degrees of freedom) gives no reason to claim that the substitution has significantly changed the goodness of fit of the model. Table I shows the estimated parameters for both models. The trend in the estimates of the age and period effects are similar for the two models. A negative coefficient of the variables of childbearing patterns means that we have a protective effect of a high value of the variable. That means that we found a significant inverse association between cancer incidence and number of children born by women 20-29 years $\left(\gamma_{2}\right)$ of age. There is also a favourable significant association between breast cancer and number of children born by the oldest women $\left(\gamma_{4}\right)$, while number of children born by women under 20 years of age and by women $30-39$ years of age showed a positive but far from significant association to breast cancer incidence.

According to the $\gamma$ estimates in model 2 we can see that among all the cohorts included in this study, the 1890-94 cohort had the most favourable childbearing pattern with regard to the risk of breast cancer, while the 1910-14 cohort had the least favourable pattern. If all the cohorts had the same childbearing pattern as the 1890-94 cohort, the estimated cumulative incidence (ie, an age adjustment according to $\mathrm{Day}^{8}$ ) for the observation period 1975-84 would have been 37.87 per 1000 women instead of 44.24 per 1000 as observed. A different way of demonstrating the 
Table I Estimated coefficients in the two models of breast cancer $195 \%$ confidence intervals are given in brackets)

\begin{tabular}{|c|c|c|c|c|c|c|c|c|}
\hline \multirow[b]{3}{*}{ Age } & \multirow{3}{*}{\multicolumn{2}{|c|}{$\begin{array}{ll}\alpha_{1}: & 35-39 \\
\alpha_{2}: & 40-44 \\
\alpha_{3}: & 45-49 \\
\alpha_{4}: & 50-54 \\
\alpha_{5}: & 55-59 \\
\alpha_{6}: & 60-64 \\
\alpha_{7}: & 65-69\end{array}$}} & \multicolumn{3}{|l|}{ Model 1} & \multicolumn{3}{|l|}{ Model 2} \\
\hline & & & \multicolumn{3}{|c|}{$-8.000(-8.088,-7.912)$} & \multicolumn{3}{|c|}{$-7.753(-7.898,-7.607)$} \\
\hline & & & $\begin{array}{l}0 \\
0.678 \\
1.051 \\
1.018 \\
1.111 \\
1.260 \\
1.364\end{array}$ & $\begin{array}{c}(-) \\
(0.601, \\
(0.974, \\
(0.940, \\
(1.027, \\
(1.176, \\
(1.267,\end{array}$ & $\begin{array}{l}0.755) \\
1.128) \\
1.096) \\
1.195) \\
1.344) \\
1.461)\end{array}$ & $\begin{array}{l}0 \\
0.621 \\
0.968 \\
1.127 \\
1.245 \\
1.413 \\
1.534\end{array}$ & $\begin{array}{c}(-) \\
(0.488, \\
(0.778, \\
(0.872, \\
(0.979, \\
(1 \cdot 139, \\
(1 \cdot 247,\end{array}$ & $\begin{array}{l}0.753) \\
1.159) \\
1.382) \\
1.500) \\
1.687) \\
1.821)\end{array}$ \\
\hline Period & $\begin{array}{l}\pi_{1}: \\
\pi_{2}: \\
\pi_{3}: \\
\pi_{4}: \\
\pi_{5}:\end{array}$ & $\begin{array}{l}1955-64 \\
1960-69 \\
1965-74 \\
1970-79 \\
1975-84\end{array}$ & $\begin{array}{l}0 \\
0.079 \\
0 \cdot 154 \\
0 \cdot 290 \\
0.305\end{array}$ & $\begin{array}{c}(-) \\
(0.031, \\
(0.100, \\
(0.236, \\
(0.251,\end{array}$ & $\begin{array}{l}0.127) \\
0.208) \\
0.344) \\
0.359)\end{array}$ & $\begin{array}{l}0 \\
0 \cdot 071 \\
0 \cdot 127 \\
0 \cdot 247 \\
0 \cdot 238\end{array}$ & $\begin{array}{c}(-) \\
(0.025, \\
(0.080, \\
(0.196, \\
(0.170,\end{array}$ & $\begin{array}{l}0 \cdot 118) \\
0 \cdot 175) \\
0 \cdot 299) \\
0 \cdot 306)\end{array}$ \\
\hline Cohort & $\begin{array}{l}\beta_{1}: \\
\beta_{2}: \\
\beta_{3}: \\
\beta_{4}: \\
\beta_{5}: \\
\beta_{6}: \\
\beta_{7}: \\
\beta_{8}: \\
\beta_{9}: \\
\beta_{10}: \\
\beta_{11}:\end{array}$ & $\begin{array}{l}1890-94 \\
1895-99 \\
1900-04 \\
1905-09 \\
1910-14 \\
1915-19 \\
1920-24 \\
1925-29 \\
1930-34 \\
1935-39 \\
1940-44\end{array}$ & $\begin{array}{l}0 \\
0.077 \\
0.031 \\
0.062 \\
0.091 \\
0.047 \\
0.049 \\
-0.011 \\
-0.070 \\
-0.056 \\
0\end{array}$ & $\begin{array}{c}(-) \\
(-0.026, \\
-0.063, \\
-0.027, \\
-0.003, \\
-0.048, \\
-0.049, \\
-0.122, \\
-0.195, \\
-0.199, \\
\text { aliased }\end{array}$ & $\begin{array}{l}0.179) \\
0.125) \\
0.152) \\
0.178) \\
0.141) \\
0.149) \\
0.101) \\
0.055) \\
0.086)\end{array}$ & & & \\
\hline $\begin{array}{l}\text { Childbearing } \\
\text { per woman }\end{array}$ & $\begin{array}{l}\gamma_{1}: \\
\gamma_{2}: \\
\gamma_{3}: \\
\gamma_{4}:\end{array}$ & & & & & $\begin{array}{r}1 \cdot 299 \\
-0.240 \\
0.106 \\
-1.414\end{array}$ & $\begin{array}{l}-0.557, \\
-0.390, \\
-0.102, \\
-2.578\end{array}$ & $\begin{array}{r}3.155) \\
-0.097) \\
0.314) \\
-0.250)\end{array}$ \\
\hline
\end{tabular}

Model 1: $\exp \left(\xi+\alpha_{j}+\pi_{k}+\beta_{j}\right)$
Model 2: $\exp \left(\xi+\alpha_{j}+\pi_{k}+\gamma_{1 \star} N C 19_{j}+\gamma_{2} \star N C 29_{j}+\gamma_{3 \star} N C 39_{j}+\gamma_{4 \star} N C 49_{j}\right)$

influence of the childbearing pattern is shown in fig 3. Here the observed cumulative incidence for the periods of diagnosis between 1955 and 1984 are compared to the estimated cumulative incidence given that the cross sectional childbearing pattern for each of the five periods of diagnosis are the same and equal to the childbearing pattern of the first period. That means that $15 \%$ of the increase in cumulative incidence from the level of 33.60 per 1000 women in the first period to 44.24 per 1000 women in the last may be explained by changes in the childbearing pattern among those under risk of getting a breast cancer.

Figure 4 shows the observed incidence of uterine corpus cancer by age and cohort. There are large differences between the different cohorts, and the shape of the curves indicates that the number of new uterine corpus cancer cases per 100000 women can be expected to increase in the future. If we do the same modelling exercise for uterine corpus cancer as for breast cancer, we find a deviance for model 1 of 13.4 with 15 degrees of

Figure 3 Observed cumulative incidence of breast cancer compared with estimated cumulative incidence given that the childbearing pattern was as for the period 1955-64.

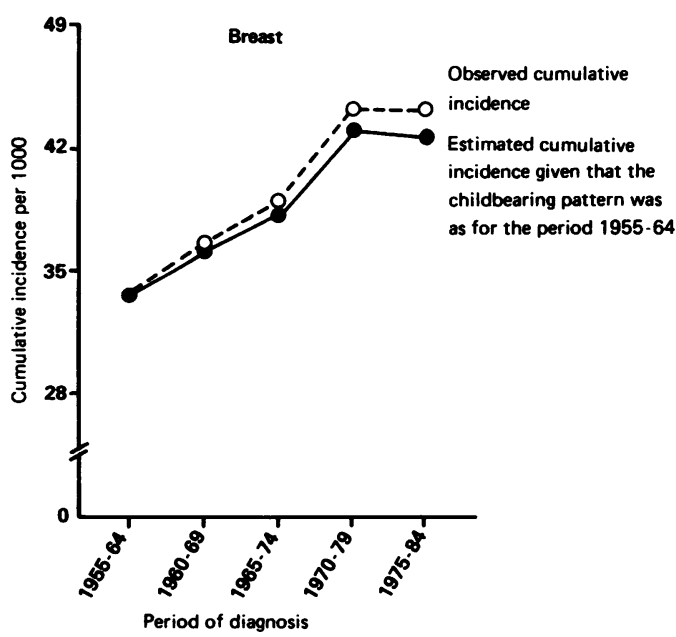

freedom, which is close to the expected value. By substituting the cohort parameters with the variables describing the childbearing pattern of the cohorts, this model (model 2) obtained a deviance of 21.6 with 20 degrees of freedom.

Table II shows the estimated parameters in both models. The shapes of the age and period effects do not occur very differently in the two models. The variables NC29 and NC49 contribute significantly in the model. The $\gamma$ values for NC29, NC39 and NC49 are negative which means that there is an inverse association between all variables and the risk of uterine corpus cancer.

From Model 2 we can see that the most favourable cohort with respect to childbearing pattern and risk of uterine corpus cancer is the 1890-94 cohort. If all later cohorts had the same childbearing pattern as this cohort, the estimated cumulative incidence of uterine corpus cancer for the observation period 1975-84 would have been $7 \cdot 21$ cases per 1000 women compared to the observed 11.83 cases per 1000 . Figure 5 shows, in the same manner as in fig 3 , the influence of changes in childbearing pattern on the cumulative incidence of uterine corpus cancer through the periods between 1955 to 1984 . Twenty seven per

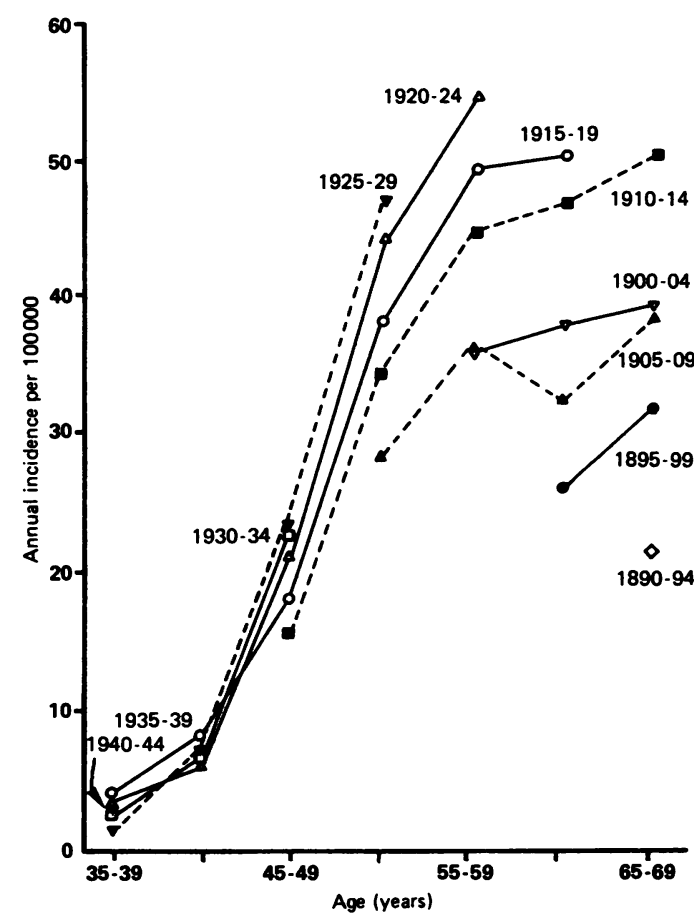

Figure 4 Incidence of uterine corpus cancer by age and birth cohort.

cent of the increases in cumulative incidence from 6.97 per 1000 women for the period 1955-64 to 11.83 per 1000 for the period 1975-84 may be attributed to changes in childbearing patterns among those under risk of getting a uterine corpus cancer in these two periods.

Discussion

To our knowledge this type of cohort analysis of breast cancer and cancer of uterine corpus has not been done before. However, the study resembles that by Stevens and Moolgavkar ${ }^{9}$ who carried out 
a cohort analysis of lung cancer and smoking. This type of study is statistical and not biological, which implies that any data connected with the cohort which vary in the same manner as our four variables describing childbearing pattern will also explain variations in cancer incidence.

It is difficult to make comparisons between the influence of our variables describing childbearing pattern for the cohorts and reproduction variables used in previous studies made on the basis of the individual. Part of the difficulty lies in the definition of the variables. In the case of breast cancer it seems favourable to have many children before the age of 30 . This agrees with most of the earlier studies. ${ }^{1} \mathrm{NC} 49$ is a variable indicating the cohort's tendency to have children late in the fertile period of life. There are not many studies concerning age at births after the first and none of them indicate a protective effect of a late last birth. However, the recent study by Kvåle and $\mathrm{Heuch}^{2}$ indicates that the relation between age when a pregnancy occurs and the risk of breast cancer is more complex than previously believed.

Table II Estimated coefficients in the two models of uterine corpus cancer $195 \%$ confidence intervals are given in brackets)

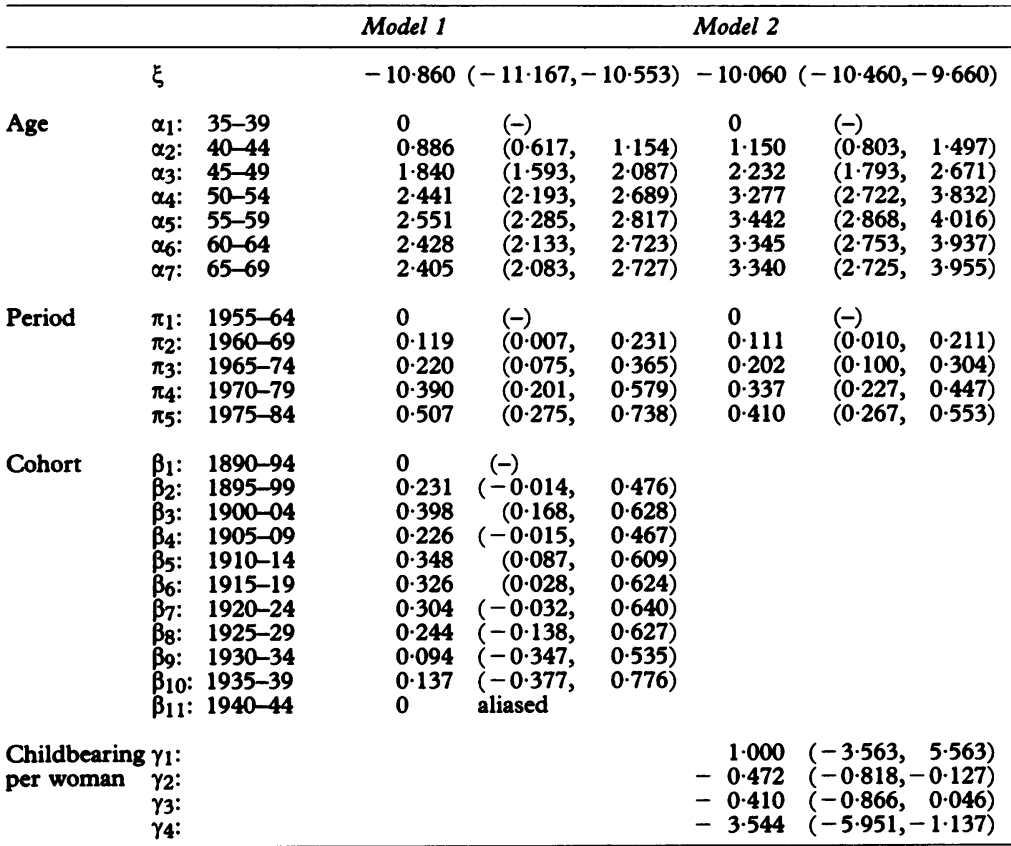

Model 1: $\exp \left(\xi+\alpha_{j}+\pi_{k}+\beta_{j}\right)$
Model 2: $\exp \left(\xi+\alpha_{j}+\pi_{k}+\gamma_{1} \star N C 19_{j}+\gamma_{2} \star N C 29_{j}+\gamma_{3} \star N C 39_{j}+\gamma_{4} \star N C 49_{j}\right)$

Figure 5 Observed cumulative incidence of uterine corpus cancer compared with estimated cumulative incidence given that the childbearing pattern was as for the period 1955-64.

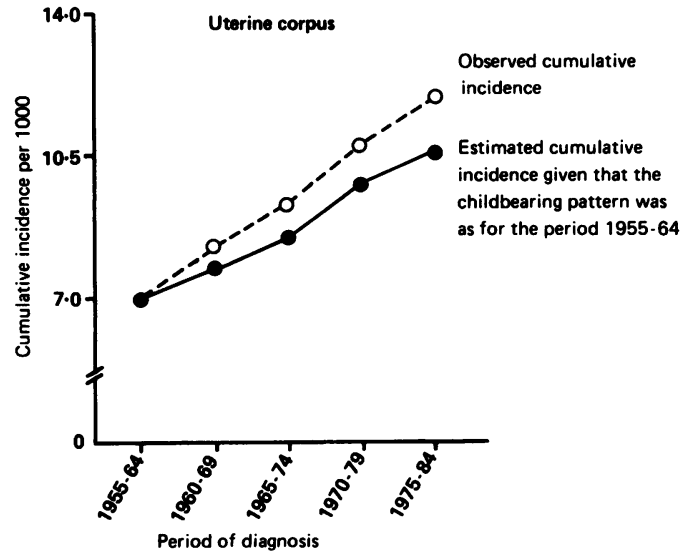

For cancer of the uterine corpus we found a significant protective effect of two of the variables, while NC39 showed a protective effect but did not reach the significance level. Only the variable given by the number of children born by the women in the cohort before 20 years of age showed a positive but non-significant association to the risk of uterine corpus cancer. This seems reasonable compared with other studies showing a favourable effect of high parity, ${ }^{410}$ and a decreasing risk with an increasing age at first and last birth. ${ }^{10}$

If we accept this type of modelling, the model tells us that about $15 \%$ of the increase in the cumulative incidence of breast cancer from 1955 to 1984 may be directly attributed to changes in childbearing pattern (fig 3). Most of the increase may be explained by the period effect which indicates changes in environmental factorsamong others dietary factors seem to be such possible factors. For example, results by $\mathrm{Hems}^{11}$ show that the variation of breast cancer mortality between 41 countries appeared to arise predominantly from differences in diet, the contribution from the variation in childbearing being small. In another study, ${ }^{12}$ he found that changes in breast cancer mortality for all women in England and Wales between 1911 and 1975 were not related to childbearing, but to changes in consumption of fat, sugar, animal protein 1-2 decades earlier. However, the same study showed, in contrast, that the geographical variation of breast cancer mortality within the United Kingdom, by region or by urban-rural aggregate area, was closely correlated with childbearing but poorly correlated with diet.

Other factors that may influence the risk of breast cancer are menarcheal age and age at menopause. While the menarcheal age has had a monotonically decreasing trend in this century in Norway, ${ }^{13}$ the age at menopause has increased by calendar time. Kvalle ${ }^{14}$ found that the correlation coefficients between reproductive factors and age at menarche or age at menopause ranged from -0.02 to 0.06 and it is unlikely that these two menstrual factors could be strong confounding factors for the associations between reproductive factors and breast cancer on the individual basis seen in their study. ${ }^{215}$ However, if the trend in NC49 by cohorts is similar to that of menarcheal age or to the inverse of the trend in age at menopause, it could be that the significant inverse association between NC49 and the risk of breast cancer would disappear if these variables were included in the analysis.

The breast cancer results are also in accordance with those by Moolgavkar et al $^{16}$ and Hems ${ }^{17}$ which indicated that differences between populations cannot be explained by factors related to childbearing pattern.

Our model indicates that $27^{\circ} \%$ of the increase in the cumulative incidence of uterine corpus cancer in the period 1955 to 1984 in Norway may be directly attributed to changes in the childbearing pattern (fig 5). On the West Coast of the United States a rise in incidence of endometrial cancer began in the middle of 1960 s and reached a peak in $1975 .{ }^{18}$ The rise was connected with the use of replacement oestrogen treatment. Such a trend in the incidence of uterine corpus cancer has not 
been noticed in Norway. Such treatment has only been used in the last decade of the observation period and even only then to a limited extent. ${ }^{19}$ If the incidence of uterine corpus cancer in Norway is influenced by the use of replacement oestrogen treatment, the effect is small and probably included in the period effect.

We do not believe that the use of hysterectomy has been an important factor in this study. In a Norwegian study covering the age group 27-69 years, 1011 out of 62079 women $(1.6 \%)$ reported surgical removal of the uterus. ${ }^{20}$ The rate of hysterectomy in Norway is much lower than the frequency observed in districts in England and in New England. ${ }^{21}$

Overweight is a strong risk factor for uterine corpus cancer. We do not know how this factor changes between the different cohorts and by calendar time. Kvalle et al $^{14}$ have shown that the correlation coefficients between body mass index (weight/height ${ }^{2}$ ) and the reproduction factors of parity and age at first and last birth were $0 \cdot 13$, -0.05 , and 0.06 respectively on an individual basis. Kvale et al concluded that a stratification by body mass index did not notably influence risk estimates for reproductive factors.

We thank Dr Ashton Miller and Mr Øystein Kravdal for valuable comments during preparation of this manuscript. Tor Haldorsen is a Fellow of the Norwegian Cancer Society.

1 Thomas DB. Epidemiology and related studies of breast cancer etiology. In: Lilleinfeld AM, ed. Reviews in cancer epidemiology. Vol 1, New York: Elsevier North Holland, epidemiology.

2 Kvale G, Heuch I. A prospective study of reproductive factors and breast cancer. II Age at first and last birth. $A m \mathcal{F}$ Epidemiol 1987; 126: 842-50.
3 Kelsey JL, LiVolsi VA, Holford TR, et al. A case control study of cancer of the endometrium. Am $\mathcal{F}$ Epidemiol 1982; 116: $333-42$.

4 Henderson BE, Caragrande JT, Pike MC, Mack T, Rosario Henderson BE, Caragrande JT, Pike MC, Mack T, Rosario I, Duke A. The epidemiology of endometrial

5 Brunborg H. Cohort and period fertility for Norway 18451985. Rapporter 88/4, Central Bureau of Statistics of Norway, 1988

6 Clayton D, Schifflers E. Models of temporal variation in cancer rates. II: the age-period-cohort models. Stat Med 1987; 6: 469-81.

7 Clemmesen J. Carcinoma of the breast. Sypmposium. Results from statistical research. $\mathrm{Br} \mathcal{F}$ Radiol 1948; 21 583-90.

8 Day NE. Cumulative rate and cumulative risk. In: Waterhouse J, Muir C, Shanmugaratnam K, Powell J, eds. Cancer incidence in five continents. Vol IV IARC Scientific Publications No 42. Lyon: International Agency for Publications No 42. Lyon: Inte

9 Stevens RG, Moolgavkar SH. A cohort analysis of lung cancer and smoking in British males. Am $\mathcal{F}$ Epidemiol 1984; cancer and smo: $624-41$.

10 Kvåle G, Heuch I, Giske U. Reproductive factors and risk of cancer of the uterine corpus: a prospective study. Cancer Research 1988; 48: 6217-22.

11 Hems $G$. The contribution of diet and childbearing to breast-cancer rates. $B r \mathcal{F}$ Cancer 1978; 37: 974-82.

12 Hems G. Association between breast-cancer mortality rates, childbearing and diet in the United Kingdom. Br $\mathcal{f}$ Cancer 1980; 41: 429-37.

13 Brudevoll JE, Liestøl K, and Walløe L. Menarcheal age in Oslo during the last 140 years. Ann Hum Biol 1979; 6: 407-16.

14 Kvale G. Reproductive factors and risk of cancers of the breast and genital organs. A prospective study of Norwegian women. and genital organs. A prospective study

15 Kvảle G, Heuch I, Eide GE. A prospective study of reproductive factors and breast cancer. I. Parity. $A m \stackrel{\mathcal{J}}{ }$ Epidemiol 1987; 126: 831-41.

16 Moolgavkar SH, Lee JAH, Hade RD. Comparison of age-specific mortality from breast cancer in males in the United States and Japan. $\mathcal{F}$ Natl Cancer Inst 1978; 60: 1223-6.

17 Hems G, Stuart A. Breast cancer rates in populations of single women. Br f Cancer 1975; 31: 118-23.

18 Jick $H$, Walker AM, Rothman KJ. The epidemic of endometrial cancer: a commentary. Am $\mathcal{f}$ Public Health 1980; 70: 264-7.

19 Bergsjo P. Comments on the use of oestrogens 1979-83. In: Drug consumption in Norway. Oslo: Norsk Medisinaldepot 1984: $114-5$.

20 Kvále G, Heuch I, Ursin G. Reproductive factors and risk of cancer of the uterine corpus. A prospective study. Cancer Research 1988; 48: 6217-2

21 McPherson K, Clifford P, Wennburg J, Hovind OB. Small area variations in the use of common surgical procedures: An international comparison of New England, England and Norway. N Engl f Med 1982; 307: 1310-4. 